\title{
Auf dem Weg zu einer metropolitanen Regulation? Der Verein Metropolitanraum Zürich
}

\author{
R. Nüssli \\ Dozentur Soziologie, Departement Architektur, ETH Zürich, Schweiz \\ Correspondence to: R. Nüssli (nuessli@arch.ethz.ch)
}

Received: 9 May 2014 - Revised: 25 July 2014 - Accepted: 11 August 2014 - Published: 26 January 2015

\begin{abstract}
Kurzfassung. The Metropolitan Region of Zurich is fragmented into eight cantons and marked by ideological cleavages. Nevertheless, the "Association Metropolitan Region Zurich" has established since 2007. The paper asks, how the metropolitan restructuring is possible. Tracing the question, first the scale-debate is opposed to the spatial governance-debate and it shall be argued that the scale-debate serves as a more precise tool of analysis. Building on this theoretical foundation the institutionalising of the association is analysed on the basis of 13 expert interviews. Three points are of particular relevance: First, precisely the fragmentation of the metropolitan region forms a crucial reason for the rapid introduction of the association. Second, the institutionalisation brings a subtle power to the association because it does not openly question the federalist system. Third, its agency manifests in territorial implications such as the revision of a national infrastructure plan or a metropolitan spatial plan, which both are made possible by simultaneous processes of informalisation and formalisation on a new scale. At the end it shall be demonstrated that the association's neoliberal agenda is hardly contested, raising the question of the scale of possible opposition.
\end{abstract}

\section{Einleitung}

Seit 2007 etabliert sich der „Verein Metropolitanraum Zürich" (VMZ) als überkantonaler Akteur, der sich um das Regieren des Urbanisierungsprozesses bemüht. Die Existenz des Vereins überrascht angesichts des stark ausgeprägten Föderalismus und markiert einen Paradigmenwechsel in der schweizerischen Urbanisierungspolitik. Sie überrascht aber auch angesichts einer polymorphen Metropolitanregion, die von zahlreichen Diskrepanzen geprägt ist. Mit dem Kanton Schwyz und dem Kanton Zug grenzen zwei der steuergünstigsten Kantone direkt an den Kanton Zürich (ESTV, 2013). Der steuertechnische Graben geht insbesondere im Fall des Kantons Schwyz einher mit ideologischen Kontrasten (s. Nüssli, 2015). Aber auch zwischen den Städten und den sogenannten Agglomerationen zeigen sich in Abstimmungen immer stärkere ideologische Gräben. Während in den Städten eine linke und weltoffene Haltung dominiert, manifestiert sich in den Agglomerationsgürteln eine rechtskonservative Einstellung (vgl. Hermann und Leuthold, 2003; Kübler et al., 2013).
In wissenschaftlichen Publikationen zeichnet sich der Paradigmenwechsel hin zu einer grossräumigeren Urbanisierungspolitik schon etwas länger $a b$. Eine der frühesten urbanen Konzeptionen der Schweiz findet sich in „Metropole Schweiz“ (Bassand und Schuler, 1985), die die Schweiz als polyzentrisches Netz sieht, das funktional eine Metropole bildet. Erst im gegenwärtigen Jahrhundert skizzieren mehrere Arbeiten ein Land, das aus verschiedenen, grenzüberschreitenden Metropolen besteht. "Stadtland Schweiz" (Eisinger und Schneider, 2003) bildet den Auftakt einer Denkfigur der Schweiz als Stadt, die den Föderalismus herausfordert. Zwei Jahre später folgt der Raumentwicklungsbericht des Bundesamts für Raumentwicklung (ARE, 2005), der von einer polyzentrischen Schweiz mit fünf Metropolen ausgeht. 2006 stellen Thierstein et al. die These einer „Raumentwicklung im Verborgenen“ auf, im Zuge derer sich ,unbemerkt" (ibid.: 14) zwei Metropolregionen herausgebildet hätten. Im gleichen Jahr bricht die Publikation „Die Schweiz Ein städtebauliches Portrait“ (Diener et al., 2006) mit der Vorstellung einer städtischen und einer ländlichen Schweiz, 
indem sie die Schweiz als vollständig urbanisiertes Territorium konzipiert, weil auch die sogenannten Peripherien längst in die Metropolitanräume eingebunden seien und deren Funktionieren wesentlich unterstützten. Das aktuelle Raumkonzept des Bundes (Schweizerischer Bundesrat, 2012) geht ebenfalls von den drei Metropolitanregionen Basel, Genf und Zürich aus.

Allerdings bescheinigen die genannten Publikationen einer grenzübergreifenden politischen Zusammenarbeit kaum Chancen (z.B. Diener et al., 2006:612). So attestiert etwa Lendi (2008:385) den sogenannten Agglomerationen eine Zukunft als ,„Pflegekinder' der vorgegebenen politischen Einheiten, also vorläufig wohl ohne eigene Governance, ohne politisches Eigengewicht". Das städtebauliche Portrait spricht im Fall von Zürich von einem „Anti-Zürich-Reflex“ und einer ,anti-urbanen Haltung“ (Diener et al., 2006:612). Die pessimistischen Prophezeiungen haben sich als falsch erwiesen. Der VMZ ist ein Exempel für einen Paradigmenwechsel in der schweizerischen Urbanisierungspolitik, die sich vermehrt den Zentren zuwendet und den ausgeprägten Föderalismus trotz ideologischen Kontrasten teilweise aufbricht.

Dieser Artikel fragt einerseits, wie es zu dieser metropolitanen Restrukturierung gekommen ist und welche Implikationen dieser Restrukturierungsprozess hat. Diese Fragen treiben neben Politikwissenschaftler_innen auch einen Teil der geografischen Forschung um, die danach fragt, ,wie, von wem und zu welchem Zweck Massstabsebenen durch soziale Praxis produziert und/oder strategisch eingesetzt werden“ (Belina, 2008:113; vgl. Schmid, 2003:222; Wissen, 2008:9; Paasi, 2010:2301). Die Frage, wie Massstabsebenen produziert werden, wurde bis anhin selten empirisch erforscht (Marston et al., 2005; Miller, 2009). Mit diesen Fragen einher geht meiner Ansicht nach eine zweite Perspektive, die sich auf widersprechende Positionen gegenüber Restrukturierungsprozessen konzentriert. Dieser zweite Blick zieht sich sowohl im theoretischen wie im empirischen Teil ebenfalls durch den Artikel.

Im internationalen wie auch im nationalen Diskurs lässt sich nach einer intensiven Auseinandersetzung ab den 1990er bis in die späten 2000er Jahre ein deutlicher Rückgang an Publikationen zu metropolitanen Restrukturierungen feststellen. Das Thema bleibt meiner Ansicht nach aber aktuell, da sich die Restrukturierungsprozesse fortsetzen und damit die Massstabsebene keinesfalls fix oder wissenschaftlich geklärt ist. Zum andern werden Fragen nach kritischen Auseinandersetzungen und Alternativen zur hegemonialen Ideologie der Metropolitanregion selten gestellt. Um den aufgeworfenen Fragen nachzugehen, führe ich im nächsten Kapitel in die wissenschaftliche Diskussion im Feld der metropolitanen Restrukturierungsprozesse ein. Ich stelle die ScaleDebatte der Debatte um Metropolitan Governance gegenüber und argumentiere, dass die Scale-Debatte das schärfere Analysewerkzeug bietet. Im Anschluss erarbeite ich zwei Paradoxe gegenwärtiger Urbanisierungspolitiken, die teilweise auch in der Governance-Forschung behandelt werden. Darauf aufbauend widmet sich das folgende Kapitel dem schweizerischen Urbanisierungsleitbild, in dessen Uneindeutigkeit sich der VMZ etabliert. Auf diesem Fundament analysieren die letzten beiden Kapitel die Institutionalisierung des VMZ anhand von Interviewmaterial. Zum Schluss wird die Frage nach Demokratisierung und Möglichkeiten emanzipatorischer Politik für den untersuchten Fall nochmals aufgenommen. Diese Frage wird in der kritischen Stadtforschung ${ }^{1}$ bis anhin fast immer auf den lokalen und/oder den globalen Massstab bezogen.

\section{Metropolitane Restrukturierungsprozesse in der theoretischen Debatte}

Mit der Frage nach der Produktion von Massstabsebenen situiert sich der Artikel in der Scale-Debatte, die in der Tradition der materialistischen Analyse Lefebvres steht und auf die räumliche Massstäblichkeit sozialer Prozesse fokussiert. Ihre Ursprünge hat sie bei Taylor (1982), Smith (1984) und Harvey (1985). In den 1990er Jahren wurde sie um die Bedeutsamkeit sozialer Kämpfe (Herod, 1991; Swyngedouw, 1997) einem relationalen, prozesshaften Massstabsverständnis (Brenner, 1998, 2004) und einer Relativierung des räumlichen Massstabs hinsichtlich einer privilegierten Ebene der Produktion von Staatlichkeit (Jessop, 2009) erweitert. Mahon und Keil (2009:8) sprechen gar von einem "scalar turn" in der Geografie.

Unter dem Begriff Scale, den ich mit Massstabsebene übersetze, verstehen etwa Leitner und Sheppard (2009:231) ,a relational, power-laden, and contested construction that actors strategically engage with in order to legitimize or challenge existing power relations. In the course of these struggles, new scales are constructed, and the relative importance of different scales is reconfigured". ${ }^{2}$ Mit dem Fokus auf die

\footnotetext{
${ }^{1}$ Kritische Stadtforschung hat sich als Begriff weit etabliert - im deutschsprachigen Raum lediglich seit einigen Jahren - und bezieht sich häufig auf die angloamerikanische Radical Geography. Die Ansage, kritische Wissenschaft zu betreiben oder sich auf solche theoretische Konzepte zu beziehen, verweist meist (implizit) auf das Theoriegebäude Marx' sowie der Frankfurter Schule und fungiert so als Codewort für eine Position, die sich gegen jegliche Form von Herrschaft und Unterdrückung wendet (Kilminster, 2013). „In short, critical urban theory involves the critique of ideology (including social-scientific ideologies) and the critique of power, inequality, injustice and exploitation, at once within and among cities." (Brenner, 2009:198). Einen deutschsprachigen Einstieg bieten Belina et al. (2014). Kemper und Vogelpohl (2013:20-24) verweisen explizit auf die Scale-Debatte als Ansatzpunkt einer kritischen Stadtforschung. Der Gewinn des Begriffs liegt für mich aber neben dem ideologischen Verweis vor allem im Anspruch an eine kritische Reflexion des Forschungsprozesses sowie der Position der Forschenden.

${ }^{2}$ Für eine Auseinandersetzung mit dem Begriff s. auch Brenner (2004:7-12); Wissen (2008); Miller (2009). Für eine Auseinander-
} 
Produktion von Massstabsebenen vernachlässigt die ScaleDebatte häufig die strukturierenden Wirkungen von Massstabsebenen und den dazugehörigen Diskursen auf das soziale Handeln (Herod und Wright, 2002:11; Wissen, 2008:20). Ich versuche deshalb bei der Analyse des VMZ eine doppelte Perspektive einzunehmen, die sowohl auf die Produktionswie auf die Verdinglichungsprozesse fokussiert.

\subsection{Metropolitaner Massstab als privilegierte Arena der Standortpolitik}

Metropolitane Restrukturierungsprozesse werden in der Fachliteratur häufig mit dem Ende des Fordismus in Zusammenhang gebracht. Während sich der ,westliche“ Nationalstaat in der fordistischen Akkumulationsphase um räumlichen Ausgleich bemühte (z.B. Castells und Godard, 1974; Lipietz, 1995:92-99), destabilisierte die zunehmende Globalisierung die politische Geografie des Nationalstaats in Mittel- und Westeuropa bereits in den 1970er Jahren (z.B. Krebs, 1996). Die sogenannte Desindustrialisierung der Zentren bewirkt ab Mitte der 1980er Jahre einen verstärkten Wettbewerb um Investitionen zwischen den Städten. Statt interregionalem räumlichem Ausgleich steht zunehmend der internationale Wettbewerb der Städte auf der Agenda (z.B. Harvey, 1989; Hall und Hubbard, 1998; McCann, 2004). Sie werden zu den „Motoren der globalen Wirtschaft“ (Scott, 1996). Die „Global Cities“ (Sassen, 1991) lösen die Nationalstaaten aber auch in ihren Herrschafts- und Kontrollfunktionen teilweise ab - gewissermassen ein Downscaling der Staatlichkeit. Doch bereits Ende der 1990er Jahre kommt es in West- und Mitteleuropa zu neuen Upscaling-Prozessen, die die metropolitane Ebene institutionalisieren. Die Globalisierung bewirkt eine Metropolisierung, im Zuge derer sich polyzentrische Konfigurationen ausbilden, die weit über die klassische Agglomeration hinausreichen (Veltz, 1996; Scott et al., 2001; Taylor, 2004; Hall und Pain, 2006; Krätke, 2007). Die Metropolitanregionen können zwar als ökonomisch integriertes - wenn auch umkämpftes - Territorium abgebildet werden, sind aber gleichzeitig institutionell fragmentiert (Storper, 1997). Kooperation zwischen verschiedenen politischen Institutionen wird notwendig, um die Wettbewerbsfähigkeit zu erhöhen: ,[T]he new metropolitan regionalism instrumentalizes intra-regional cooperation in order to intensify the process of interspatial competition at supraregional scales." (Brenner, 2004:286)

Einerseits sind Rescaling-Prozesse also von deterritorialisierenden Momenten geprägt. Anderseits transformieren die globalen Urbanisierungsprozesse die territoriale Regulation, was Harvey (1985:145) treffend auf den Punkt brachte: ,spatial organization is necessary to overcome space“. Auf Harveys Analyse aufbauend führt Brenner (1998) die Dialektik von Deterritorialisierung und Reterritorialisierung ein: Po-

setzung über Lefebvres Verständnis von Massstabsebenen s. Kipfer (2008) litics of Scale dekonstruieren nicht ausschliesslich die bestehenden räumlichen Ebenen von sozialen, politischen und ökonomischen Beziehungen, sondern schaffen gleichzeitig eine neue geografische Fixiertheit und veränderte Geografien der Macht. Swyngedouw (1992, 1997) nimmt mit dem Begriff Glocalization das Konzept einer zunehmenden Globalisierung und einer damit einhergehenden Umstrukturierung des lokalen Territoriums auf. ${ }^{3}$ Es weist darauf hin, dass verschiedene räumliche Ebenen ineinander greifen und sich gegenseitig beeinflussen: „In short, the local and the global are mutually constituted (...).“ (Swyngedouw, 1997:137). Gemeinsam ist diesen Konzepten die Annahme, dass die zunehmende Globalisierung der Kapitalflüsse die Bedeutung der Grenzen nicht auflöst, sondern die Territorialität transformiert, da die globale Dimension der Urbanisierungsprozesse eine Rekonfiguration der bestehenden Regierungsebenen erfordere. Politics of Scale versuchen demnach, Standortvorteile gegenüber anderen urbanen Konfigurationen zu schaffen. Die Stadt als Regierungsebene wird zusehends aufgehoben. An ihre Stelle tritt - so die These - die Metropolitanregion. Neue Arrangements wie beispielsweise der VMZ können als Versuche gelesen werden, die Urbanisierung auf einer anderen Massstabsebene zu regulieren, indem sie einen neuen, temporären Scalar Fix (Smith, 1995) schaffen.

Die ökonomisch argumentierende Strömung innerhalb der kritischen Stadtforschung leitet den wesentlichen Grund der Restrukturierung also als wirtschaftlichen Zwang der kapitalistischen Urbanisierung ab. Politics of Scale werden als Versuche gelesen, sich den ständig transformierenden Geografien des Kapitals anzupassen. Die dabei entstehenden metropolitanen Arrangements basieren auf Kooperation um im Standortwettbewerb zu bestehen. Insbesondere Smith und Swingedouw haben diese Perspektive erweitert. Mit dem Begriff jumping scales betont Smith (1993), dass die Rekonfiguration von räumlichen Ebenen die Geografie der Macht verschiebt, indem einige ihre Macht und Kontrolle stärken, während andere entmächtigt werden (s. auch Swyngedouw, 1989; Jonas, 1994; Peck, 2002). Swyngedouw (1997:142) schlägt deshalb vor: „I would, in sum, advocate the abolition of the ,global' and the ,local' as conceptual tools and suggest a concentration on the politics of scale and their metaphorical and material production and transformation." Mit dem Fokus auf die Politics of Scale wird der Bezug auf eine bestimmte Massstabsebene auch zu einer diskursiven Strategie, die verschiedenen ideologischen Positionen zugeordnet werden kann (ibid.: 140). Die Massstabsebenen sind daher umkämpft: „Scale becomes the arena and moment, both discursively and materially, where sociospatial power relations are contested and compromises are negotiated and regulated. Scale, therefore, is both the result and the outcome of social struggle for power and control." (ibid.; vgl. Brenner, 2004:299). Damit handelt es sich bei der Analy-

\footnotetext{
${ }^{3}$ In eine ähnliche Richtung geht der Begriff Glurbanization (Jessop und Sum, 2000).
} 
se um Auf- oder Abwertungen von Massstabsebenen immer auch um Machtfragen (z.B. Wissen, 2008:9), weshalb es für die Untersuchung von Rescaling-Prozessen meines Erachtens entscheidend ist, auch gegenhegemoniale Positionen in den Blick zu nehmen. Einen Ansatz dazu bieten die neueren Theorien des Politischen, die weiter unten eingeführt werden.

\subsection{Metropolitan Governance als neue Regierungsform?}

Die Scale-Debatte wird im deutschsprachigen Raum bis heute wenig beachtet. Der Sammelband „Politics of Scale“ (Wissen et al., 2008) bietet einen seltenen und guten Überblick. Mit Blick auf Fragen der Kooperation und neuer Arrangements findet die Debatte aber Anschluss an die meist weit weniger kapitalismuskritisch geführte räumliche Governance-Debatte. Wie auch im englischen Sprachraum wird damit grundsätzlich eine Transformation von Government zu Governance betont. Diese Begriffsverschiebung beschreibt einen Perspektivenwechsel auf die Staatstätigkeit, wonach staatliche Behörden nicht mehr im Alleingang agieren und regieren, sondern mit verschiedenen staatlichen und nichtstaatlichen Akteur_innen kooperieren. Kooperative Formen von Urbanisierungspolitik als „neu“ zu bezeichnen, ist jedoch übertrieben. Es ist eher so, dass die stark etatistische Planung während des Fordismus ein zeitlich und räumlich begrenztes Phänomen ist (Somerville, 2005; Etzemüller, 2009; Leendertz, 2009; Altrock et al., 2012). Urban Governance in dem Sinn gab es schon lange vor der Erfindung des Begriffs.

Die Governance-Debatte ist meiner Meinung nach jedoch von einigen analytischen Unzulänglichkeiten gekennzeichnet. In der deutschsprachigen Forschung zu Governance existieren verschiedene räumliche Foki, die feinsäuberlich auseinander gehalten werden: Urban Governance (z.B. Einig et al., 2005; Sack, 2012) bezieht sich auf lokales Regieren, meist auf Ebene der administrativ abgegrenzten Stadt oder des Quartiers. Forschungsarbeiten zu Urban Governance fokussieren teilweise auch auf Beteiligungsformen der Zivilgesellschaft im Sinn von „engagierten Bürgern“ (Benz und Dose, 2010:29), während eine solche Perspektive beim Konzept einer Regional Governance (z.B. Fürst, 2010; Sturm und Bauer, 2010) meist komplett wegfällt. Letzteres wird eingesetzt um (staatliche) Institutionalisierungsprozesse auf regionaler Ebene zu analysieren. Um ähnliche Fragen nach der politischen Handlungskoordination dreht sich auch die Debatte zu Metropolitan Governance (z.B. Heinelt und Kübler, 2005; Zimmermann und Heinelt, 2012). Der Unterschied zu Regional Governance liegt vor allem im grossräumigeren Betrachtungsmassstab. Hinzu kommt die Multilevel Governance (z.B. Brunnengräber und Walk, 2007; Benz, 2010), die mit Blick auf die Massstabsfrage ein Querschnittthema darstellt. Hier steht die Verteilung staatlicher Kompetenzen über mehrere Massstabsebenen im Zentrum. Die For- schung dazu bezieht sich meist auf die Europäische Union. Governance-Analysen sind von einem staatszentrischen Ansatz gekennzeichnet (Jessop, 2004:74, 2009:99-102), der die Akteur_innen und Institutionen als interdependent konzipiert und den Koordinationsbedarf untersucht (Wissen, 2008:1112). Das Erkenntnisinteresse der Governance-Ansätze ist stark problemlösungsorientiert ausgerichtet und kümmert sich wenig um Fragen der Macht, die in Rescaling-Prozessen essentiell sind (Wissen, 2007).

Während in der anglophonen Debatte Urban Governance als relativ weites Konzept verstanden wird, auf das auch kritische Stadtforscher_innen häufig Bezug nehmen, wird im deutschsprachigen Raum zwischen verschiedenen Massstäben unterschieden. Zwar informieren sich die verschiedenen Konzepte durchaus, aber die Differenzierung bringt meiner Meinung nach mehr Konfusion und Grabenkämpfe mit sich als analytische Klarheit. Denn ein Konzept, das eine bestimmte Massstabsebene für die Analyse voraussetzt und deren Thesen nur auf diesem Massstab angewendet werden können, verhindert eine transskalare Forschung. Damit unterbindet das deutschsprachige Governance-Konzept auch einen relationalen Zugang zu Raum, der ,space as an open and ongoing production" (Massey, 2005:55) versteht, statt als fixes Territorium. Der Vorteil des Rescaling-Konzepts liegt darin, dass es keine Unterscheidung in gegebene Massstabsebenen macht, sondern die Prozesse der Produktion dieser räumlichen Ebenen in den Fokus nimmt. Während in der anglophonen Forschungstradition Urban Governance durchaus mit solchen Prozessen verknüpft wird (z.B. Brenner, 2004), versucht ein grosser Teil der deutschsprachigen Raumwissenschaften starre Grenzen aufrecht zu erhalten, die jedoch nicht fix sind, sondern immer wieder reproduziert und verändert werden. Zudem ist der staatliche Raum längst nicht mehr in klare Ebenen unterscheidbar, die unabhängig voneinander funktionieren, sondern ein Mosaik von relationalen, interskalaren Hierarchien (Lefebvre, 1991 [1974]:8588). Mit der Unterscheidung verschiedener Massstabsebenen werden Grenzen konstruiert, die nur sehr ungenau definiert werden können, wie etwa der fluide Begriff der (Metropolitan)Region zeigt (Belina, 2008; Paasi, 2010). Darüber hinaus werden diese Grenzen von Urbanisierungsprozessen ständig aufgehoben und transformiert. Der Kritikpunkt statischer und gegebener Massstabsebenen kann auf viele anglophone Governance-Arbeiten übertragen werden (Wissen, 2008:12).

Zwei weitere Probleme des Governance-Konzepts treffen auch auf die anglophone Forschung zu. Erstens existieren sehr unterschiedliche Auffassungen des GovernanceBegriffs, die von einem minimalen Staat, einer guten Regierungsführung, bis hin zu selbstorganisierten Netzwerken reichen (z.B. Rhodes, 1997; Einig et al., 2005). Zweitens ist die Anwendung des Konzepts als Analysewerkzeug kaum definiert. Insgesamt wird das Konzept inkonsistent gebraucht und stark dem jeweiligen Untersuchungsgegenstand angepasst (Füller und Marquardt, 2009:95). Mit der neuen Be- 
grifflichkeit der Governance-Debatte einher geht aber eine analytische Verschiebung, die den Blick für die jeweilige spezifische Form zeitgenössischer Urbanisierungspolitiken schärft, weil sie auch informelle, nichthierarchische Arrangements untersucht, statt ausschliesslich auf institutionalisierte und autorisierte Akteur_innen zu fokussieren (Mayntz, 2004; Rosenau, 2004).

\subsection{Zwei Paradoxe in gegenwärtigen Politiken der Urbanisierung}

Ohne in der Theorie bereits Grenzen zwischen verschiedenen Massstabsebenen und entsprechend unterschiedlichen Politikformen herzustellen, können meiner Ansicht nach zwei Paradoxe, auf die auch die Governance-Debatte hinweist, fruchtbar eingesetzt und mit der Scale-Debatte kombiniert werden. Erstens sind die Governance-Arrangements von einem hohen Grad an Flexibilität gekennzeichnet, da die Aushandlungen häufig ausserhalb der traditionellen politischen Strukturen stattfinden (Mayer, 1995:128). Die Informalität ermöglicht den Akteur_innen, Probleme ohne parteipolitischen Konkurrenzkampf zu diskutieren (Fürst, 2010:55). Zudem erlaubt kollektives Handeln auch die Verwischung von Verantwortlichkeit. ,Je besser solche Governance-Muster in der Lage sind, an den Institutionen vorbei Probleme zu bearbeiten, desto häufiger werden sie dafür genutzt." (ibid.: 58). Nichtsdestotrotz finden die Aushandlungen im „Schatten der Hierarchie“ (Mayntz und Scharpf, 1995:28) statt, denn auch kooperative Verhandlungen setzen eine demokratisch legitimierte und zur hierarchischen Intervention fähige staatliche Macht voraus und finden erst durch sie statt. „What we observe today is not a diminishment or reduction of state sovereignty and planning capacities, but a displacement from formal to informal techniques of government (...)." (Lemke, 2002:58). Mit dem Begriff Meta-Governance betont Jessop (1998:42) die Rolle des Staats, der nicht einfach das letzte Wort hat, aber strategisch den Kontext schafft, in welchem die Heterarchien geformt werden können. Die Bedeutung gemeinsamer Visionen und Diskurse ist dabei nicht zu unterschätzen.

Das zweite Paradox schliesst direkt an das erste an, indem es ebenfalls zur ,effizienteren Problembearbeitung“ beiträgt. Kooperative Verhandlungen werden häufig als Demokratisierung des Entscheidungsprozesses dargestellt, insbesondere wenn nichtstaatliche Akteur_innen in das Arrangement einbezogen werden (z.B. Einig et al., 2005:IV, für eine kritische Auseinandersetzung z.B. Mayntz, 2004). In neueren Theorien des Politischen, die erst langsam den deutschsprachigen Forschungsraum erreichen, werden solche Demokratisierungsversuche aber als ,post-politisch“ bezeichnet (Rancière, 1999; Swyngedouw, 2007; s. auch Dzudzek, 2013; Gomes de Matos, 2013; Rosemann, 2013; Rosol, 2014). Sie postulieren gegenteilige Effekte, die aus der proklamierten Öffnung der Arrangements entstehen. Da die Teilnahme an den informellen Verhandlungen von organi- sierten sowie unorganisierten nichtstaatlichen Akteur_innen weder demokratisch legitimiert noch repräsentativ ist, führten solche Arrangements oft zur weiteren Ermächtigung der besser gestellten Bevölkerungsschichten. Die Informalisierung der Beteiligung produziere,,a particular imagination of the urban in line with the demands, dreams, and aspirations of the included, while marginalized or otherwise excluded groups remain symptomatically absent" (Swyngedouw et al., 2004:214). Privatisierung, Expertisierung und Informalisierung tragen zu Politiken der Urbanisierung bei, die genuin politische Fragen einer öffentlichen Auseinandersetzung entziehen und stattdessen von legitimisierten Akteur_innen verhandelt werden.

Da solche ,post-politische“ Arrangements häufig ein demokratisches Image haben und es weder über die Beteiligungsform noch über die grundsätzlichen Ziele zu öffentlichen Aushandlungen kommt, werden gemäss den Vertreter_innen der post-politischen These selten widersprechende Positionen geäussert. Zwar kommt es zu Konflikten innerhalb des Arrangements, aber die grundsätzliche Agenda des neoliberalen Standortwettbewerbs wird von den Akteur_innen geteilt: „Post-politics rejects ideological divisions and the explicit universalization of particular political demands. Instead, the post-political condition is one in which a consensus has been built around the inevitability of neoliberal capitalism as an economic system, parliamentary democracy as the political ideal, humanitarianism and inclusive cosmopolitanism as a moral foundation." (Swyngedouw, 2010:26-27). Dieser Analyse zufolge teilen die inkludierten Akteur_innen also etwa das Ziel einer wettbewerbsfähigen Metropolitanregion. Rancière weist darauf hin, dass Diskurse und Praktiken, die sich dieser Ideologie entgegenstellen vom hegemonialen Dispositiv - in seinen Worten die Polizei - bloss als Noise dargestellt werden: ,it [the police] is an order of the visible and the sayable that sees that a particular activity is visible and another is not, that this speech is understood as discourse and another as noise." (Rancière, 1999:29). Damit wird Exklusion unsichtbar gemacht (ibid. 116). So können aus dieser Perspektive Aufstände wie 2005 in der Pariser Banlieue Clichy-sous-Bois oder 2011 im Londoner Stadtteil Tottenham, die sich beide auf zahlreiche weitere Städte ausgeweitet haben, als Protestform gelesen werden, denen das hegemoniale Dispositiv jegliche Gültigkeit abspricht (z.B. Žižek, 2007; Dzudzek und Müller, 2013).

\section{Konzeptioneller Bezugsrahmen, Methodik und Feld}

Diesem Artikel liegt nicht nur hinsichtlich der Urbanisierungsprozesse die Arbeit Lefebvres zu Grunde, sondern auch hinsichtlich der gesellschaftlichen Produktion des Raums (Lefebvre, 1991 [1974]). Diese trialektische Konzeption vereinigt die materielle Produktion (Espace perçu), die Produktion von Wissen (Espace conçu) und die Produktion von 
Bedeutungen (Espace vécu). Der Espace perçu enthält die räumliche Praxis, der Espace conçu die Repräsentationen des Raums und der Espace vécu den gelebten Raum. Im Vergleich zu den meist sehr ökonomisch argumentierenden Rescaling-Theorien führt Lefebvre die materielle Dimension mit der mentalen und sozialen Dimension des Raums zusammen. Die Produktionsprozesse sind dialektisch miteinander verbunden und schliessen sich gegenseitig ein (Schmid, 2005:226-231). Den Raum als soziales Produkt zu verstehen, macht ihn damit auch zum Ausdruck von Machtverhältnissen. Gemäss Lefebvre (1991, [1974]:11, 281, 411) unterteilt jede politische Macht den Raum entsprechend, nimmt ihre eigene Klassifizierung von Diskursen über Räume sowie Dinge und Menschen in diesen Räumen vor und dient somit der Durchsetzung und Festigung gesellschaftlicher Interessen. Unter einer solchen Perspektive sind auch die Massstabsebenen und territorialen Grenzen als gesellschaftliche (Re)Produktionsprozesse zu verstehen (Brenner, 2004:8) und Ausdruck von Machtverhältnissen. Diese konzeptionelle Verortung ermöglicht die Kombination von materiellen und diskursiven, wie auch relationalen und territorialen Raumproduktionen (zu Letzterem vgl. McCann und Ward, 2010).

Um die Produktion des Raums und damit auch der territorialen Grenzen aufzuzeigen und deren Fixiertheit aufzubrechen, fokussiere ich auf Prozesse der Regulation der Urbanisierung und der Raumproduktion (z.B. Brenner, 2004:74-77; Heeg, 2001; Schmid, 2003; Scheuplein, 2008). Mit Lefebvre vollzieht die Analyse einen perspektivischen Wechsel vom Objekt zum Prozess (Schmid, 2005:204). So inspirierend das Werk Lefebvres für die Stadtforschung ist, es bleibt doch konfus und gibt keine empirische Anleitung. Der vorliegende Artikel versucht, ein dialektisches Theorieverständnis in eine dialektische, dynamische Analyse umzusetzen. Aufbauend auf der These der vollständigen Urbanisierung (Lefebvre, 1972 [1970]), die nicht nur auf die Schweiz (Schmid, 2006), sondern auch auf den gesamten Globus (Brenner und Schmid, 2014) angewendet werden kann, spreche ich im Folgenden von Politiken der Urbanisierung statt von Stadtentwicklungspolitik. Diese Begrifflichkeit betont erstens die Prozesshaftigkeit der Raumproduktion. Zweitens vermeidet sie eine Klassifizierung in administrative Einheiten wie „die Stadt" und umgeht damit auch eine oft mitschwingende Unterteilung in urbane, suburbane und rurale Räume. Der Plural Politiken verweist auf die Vielgestaltigkeit und mögliche Widersprüchlichkeit der Prozesse, aber auch auf die Machtverhältnisse.

Die räumliche Definition einer Metropolitanregion wird in Bezug auf die Schweiz sehr unterschiedlich gehandhabt. Der Bund definiert die Metropolitanregionen statistisch, wie das auch diverse wissenschaftliche Arbeiten machen. Massgebend sind meist Bevölkerungszahl, Pendler_innenbewegungen und/oder ökonomische Parameter wie die Wertschöpfung pro Arbeitsplatz (z.B. Bassand, 2004; Schuler et al., 2005:77; Blöchlinger, 2005; Thierstein et al.,
2006). Solche statistische Definitionen sind problematisch, da sie ein Artefakt - etwa die Stadt oder die Metropolitanregion - produzieren (Brenner und Schmid, 2014). Zudem werden solche Definitionen oft bewusst grossräumig abgegrenzt, damit die Metropolitanregion in internationalen Rankings und damit im Standortwettbewerb sichtbar wird (Schmid, 2007:68). Das städtebauliche Portrait der Schweiz überlagert verschiedene quantitative und qualitative Aspekte, die stärker auf alltagsweltliche Reichweiten fokussieren (Schmid, 2006:193-200). Allerdings bleibt auch diese Abgrenzung relativ unbestimmt und wenig nachvollziehbar. Insgesamt verdeutlichen die verschiedenen Definitionen vor allem, wie schwierig es ist, das Städtische qualitativ und quantitativ zu bestimmen. Für die vorliegende Arbeit erübrigt sich die Entwicklung einer Definition, weil die Analyse auf den VMZ fokussiert, der selber eine Raumproduktion herstellt. Ich verwende diese Abgrenzung, weil hier die Wirkungsmacht des Vereins im Zentrum steht.

Das Datenmaterial besteht neben schriftlichen Quellen aus 13 semistrukturierten Leitfadeninterviews (ca. 1 Stunde) mit Expert_innen aus kantonalen und nationalen Planungsämtern, Landschaftsschutzorganisationen, Verkehrsbetrieben und Planungsbüros, die ich hauptsächlich im Jahr 2012 interviewt habe. Unter Expert_innen verstehe ich Personen, die ,über ein spezifisches Rollenwissen verfügen, solches zugeschrieben bekommen und diese besondere Kompetenz für sich selbst in Anspruch nehmen" (Przyborski und WohlrabSahr, 2009:132). In diesem Artikel interessiert aber auch ihre Deutungsmacht und die Beteiligung an der Etablierung von Deutungen (ibid.: 133). Das Material gibt hauptsächlich Auskunft über die Produktion des Espace conçu, da ich vornehmlich Expert_innen interviewt habe, die in der Dimension der Repräsentationen des (Metropolitan)Raums arbeiten. Die Auswahl der Akteur_innen erfolgte einerseits aufgrund deren Involvierung in den Verein Metropolitanraum Zürich oder aufgrund einer Organisationszugehörigkeit, die sich kritisch zur metropolitanen Restrukturierung stellt. Dieses zweite Sampling habe ich vorgenommen, um auch Narrative analysieren zu können, die nicht Teil des Regierungsarrangements sind und von diesem eventuell als Noise dargestellt werden. Es soll einen empirischen Blindfleck aufheben. Die zweite Samplingstrategie gestaltete sich allerdings schwierig, da sich, wie unten erläutert wird, kaum widersprechende Positionen finden.

Ich habe die Interviews vollständig transkribiert und dabei vom Schweizerdeutschen ins Schriftdeutsche übersetzt. Die Auswertung erfolgte entlang der Prinzipien der Grounded Theory (Strauss und Corbin, 1996 [1990]). Für diesen Artikel habe ich Zitate ausgewählt, die einen bestimmten Aspekt einer Kategorie besonders deutlich zeigen. 
4 Das schweizerische Urbanisierungsleitbild: Gleichzeitigkeit von dezentraler Besiedlung und Agglomerationsprogrammen

Der schweizerische Planungsdiskurs ist bis in die 1950er Jahre durch Rückständigkeit geprägt, der den „Widerspruch zwischen dem Leitbild einer, ländlichen' Siedlungsstruktur und der realen Entwicklung der Umlagerung der Bevölkerung in die städtischen Agglomerationen (...) nicht thematisiert" (Blanc, 1994:80). Im Zuge des helvetischen Fordismus ändert sich das Leitbild in den 1960er Jahren hin zu einem für diese Zeit typischen Anstreben des räumlichen Ausgleichs. Noch heute findet sich die ,angemessene Dezentralisation der Besiedlung und der Wirtschaft" im Artikel 1 des schweizerischen Raumplanungsgesetzes (RPG). Die Furcht vor grossen urbanen Räumen hält zumindest im Gesetz an (vgl. Eisinger, 2004:55-59). Diese Angst manifestiert sich auch in den räumlichen Aufteilungen der Regierungskonferenzen, die als Plattform für den interkantonalen Austausch dienen, um eine koordiniertere Interessenvertretung gegenüber dem Bund zu fördern. Der Kanton Zürich ist in keiner Regierungskonferenz Mitglied. Ein Interviewpartner bezeichnet die Aufteilung als „,bewusste Zersplitterung“. Diese Zersplitterung sei typisch für die Strategie des Bundes, die nach wie vor stark auf die polyzentrale Entwicklung setzt, „dass nicht die Grossen immer noch grösser werden“ (Interview 3, 2012). Entgegen der Zeitdiagnose, dass in postfordistischen Staaten räumlicher Ausgleich zugunsten der Wettbewerbsfähigkeit aufgegeben wird, weist die Schweiz nach wie vor eine hohe regionale Umverteilung auf, die strukturschwache Regionen stützen soll und gibt besonders für den sozialen Ausgleich deutlich mehr aus als die übrigen OECDStaaten (Blöchlinger, 2005:107). Dank der föderalistischen Politik wies die Schweiz 2002 auf nationaler Ebene die geringste regionalwirtschaftliche Disparität unter den OECDStaaten auf (ibid: 111). Dieser ausgeglichene Durchschnitt geht auf den interkantonalen Finanzausgleich zurück, der die starken wirtschaftlichen Disparitäten zwischen den Kantonen mindert.

Gleichzeitig sind Metropolitanregionen seit dem neuen Raumentwicklungskonzept zumindest anerkannte Realität die Versuche, das Wachstum auf metropolitaner Ebene per Gesetz zu regulieren, bleiben aber schwach. Lediglich die vom Bundesamt für Raumentwicklung (ARE) 2002, bzw. 2008 eingeführten Instrumente der Modellvorhaben und der Agglomerationsprogramme fördern die interkommunale und interkantonale Zusammenarbeit mit fachlicher, bzw. finanzieller Unterstützung.

Die Instrumente und Ziele des ARE sind also konfliktiv. Einerseits soll die Raumentwicklung nach wie vor in eine dezentrale Richtung verlaufen, anderseits wird mit dem Leitbild der „Stadtentwicklung nach Innen“ die grenzüberschreitende Zusammenarbeit gefördert und damit auch metropolitane Restrukturierungen unterstützt. Dennoch bleibt der ausgeprägte Föderalismus bestimmend. Die schweizeri- sche Raumplanung wird denn in der Fachliteratur auch für ihr wenig proaktives Handeln kritisiert. Eisinger (2004:148) spricht von „,verstädterten Landschaften“, die „unintendierte[s] Ergebnis der rapiden gesellschaftlichen Dynamik der letzten Jahrzehnte“ sind, aber dennoch ,alle Eigenschaften vermissen, die lebendige Stadträume ausmachen“. KollSchretzenmayr (2008:85) weist darauf hin, dass der Begriff Raumplanung im schweizerischen Kontext einen falschen Eindruck vermittelt: „Raumplanung ist vom Begriff her ,aktiv " - sie will gestalten, verändern. Historisch hat sie sich aber entwickelt als Reaktion auf eine unerwünschte Bauentwicklung und Abwehr von Gefahren (...).“

Die Metropolitanregionen verbleiben bis anhin eine Konstruktion ohne direkte politische Macht. Das RPG erklärt die Raumplanung im Wesentlichen zur kantonalen Aufgabe, womit die Kantone, abgesehen von wenigen nationalen Interessen, vollständige Autonomie erhalten. Die Raumplanung ist damit ein politisches Feld, das sich dem Nationalstaat fast gänzlich entzieht. Unterschiedliche Selbstverständnisse und wirtschaftliche Strategien der kantonalen Regierungen führten zu völlig unterschiedlichen Interpretationen des RPG. In den letzten Jahren wurden einige räumliche Konsequenzen dieser laxen nationalen Regierungsfähigkeit politisiert. Mit der Annahme der Zweitwohnungsinitiative 2012 und der Revision des RPG 2013 werden die Forderungen nach einer Beschränkung der Ausweitung der Bauzonen auf nationaler Ebene gestärkt.

Angesichts der stark föderalistischen Ausgestaltung der staatlichen Raumplanungspolitik und den pessimistischen wissenschaftlichen Analysen scheint es umso überraschender, dass seit der Jahrtausendwende zahlreiche überkommunale Verbünde entstanden sind. Ebenfalls Zeichen eines Aufbrechens der Kleinräumigkeit sind die ab 1990 wieder vermehrten Gemeindefusionen, zuvor seit dem zweiten Weltkrieg ein Tabu. Zwischen 1990 und 2014 hat sich die Anzahl der Gemeinden um 22 Prozent reduziert (BFS, 2014, eigene Berechnung). Auf überkantonaler Ebene bemühen sich neben dem hier untersuchten Fall etwa die „Hauptstadtregion Schweiz“ (2011 gegründet), ,metrobasel“ (2008 gegründet) und „Métropole lémanique“ (2011 gegründet) um abgestimmtere Politiken der Urbanisierung. Allerdings kennt die Schweiz keine institutionalisierte metropolitane Regierungsebene wie es etwa in Deutschland, England, Frankreich und den Niederlanden der Fall ist (Brenner, 2004; Blöchliger, 2005; Kübler, 2012). Einer solchen Institutionalisierung stehen die grosse Autonomie der Kantone sowie die nach wie vor starke Tradition des räumlichen Ausgleichs gegenüber. Für die Schweiz lässt sich der postulierte Wechsel vom dezentralisierenden räumlichen Ausgleich hin zum zentralisierenden Standortwettbewerb auf Gesetzesebene nur bedingt nachvollziehen. 


\section{Institutionalisierung des Vereins Metropolitanraum Zürich}

In diesen widersprüchlichen Planungsideologien etabliert sich der VMZ in der durch administrative Grenzen zerschnittenen Metropolitanregion Zürich als neuer Akteur, indem er eine metropolitane Massstabsebene produziert. Seit 2007 finden im Halbjahresrhythmus Metropolitankonferenzen statt, die auf Initiative der Städte Winterthur und Zürich sowie des Kantons Zürich entstanden und durch ein Modellvorhaben des ARE unterstützt wurden. ${ }^{4} 2009$ gründet sich der Verein Metropolitanraum Zürich. Der Zweck des Vereins (Statuten, Art. 2) ist es, ,einen Beitrag zur (...) Lebensqualität (...) und zur internationalen Wettbewerbsfähigkeit“ zu leisten, ,Projekte zur Stärkung des Metropolitanraums gemeinsam anzugehen“ und „Verbindungen zu anderen Metropolitanräumen“ zu pflegen. Mitte 2014 zählte der Verein 110 Mitgliedergemeinden. Die Gemeinden der Metropolitanregion können mit 420 Schweizer Franken pro 4000 Einwohner_innen Mitglied werden. Die Mitgliedschaft ermöglicht insbesondere das nach Einwohnerstärke gewichtete Abstimmen an den Metropolitankonferenzen. Die Gemeinden werden durch gewählte Exekutivpolitiker_innen vertreten. Diese wählen auch den Metropolitanrat, der Projektteams einsetzen kann um spezifische Fragen auszuarbeiten. Daraus entstand etwa das „Metrobild“, ein metropolitanes Raumentwicklungskonzept, das weiter unten genauer vorgestellt wird.

Der Verein wurde bis anhin erst von wenigen Wissenschaftler_innen besprochen (Kübler, 2013), wie auch generell metropolitanes Rescaling in der Schweiz selten rezipiert wird (Behrendt und Kruse, 2001; Schmid, 2007; Kübler und Scheuss, 2007; Kübler und Koch, 2008; Plüss, 2008; van der Heiden et al., 2013).

\subsection{Starkes Zentrum und Zerschnittenheit der Metropolitanregion als Grundlage}

Zur schnellen Etablierung des VMZ tragen meiner Ansicht nach insbesondere drei Punkte bei. Erstens hat sich, ausgelöst durch das starke ökonomische Wachstum der Metropolitanregion sowie die Zunahme an Arbeitsplätzen (Kuster und Meier, 2008:9) und der damit einhergehenden fortschreitenden Urbanisierung, der diskursiv hergestellte Problemdruck erhöht und zwar in weiten Teilen der Metropolitanregion. Die zunehmende Mobilität verstärkt die Beziehungen zwischen verschiedenen Zentren und Peripherien zusätzlich, so dass von einem funktional integrierten Raum gesprochen werden kann. Der VMZ ist ein Versuch, den proklamierten gemeinsamen Herausforderungen zu begegnen.

Das Bewusstsein dieser Veränderungen wird zweitens von vielen Akteur_innen in ihrem Diskurs über die Urbanisie-

\footnotetext{
${ }^{4}$ Für eine ausführlichere Darstellung zur Genese und der Struktur des Vereins s. Kübler (2013).
}

rung aufgenommen. Diese Änderung des Diskurses äussert sich auch in einer Änderung der Praxis:

Ich bewerte die Zusammenarbeit bei Weitem nicht so pessimistisch, wie sie häufig dargestellt wird. Es reift schrittweise ein Erkenntnisprozess. Zwar ändern sich mal Zuständigkeiten oder es kommt zu einem personellen Wechsel, was die Zusammenarbeit manchmal erschweren kann, aber in der Grundtendenz ist die Zusammenarbeit besser geworden. Davon bin ich überzeugt. Und wenn man beispielsweise beim Agglomerationsprogramm oder dem regionalen Richtplan ${ }^{5}$ den Gedanken der Zusammenarbeit konsequent durchzieht, dann verfestigt sich das und man kommt weg von Einzelentscheidungen, die an der Gemeindegrenze aufhören. (Interview 3, 2012)

Diese Erfahrungen, die Diskurse und Praktiken ändern und Zusammenarbeit als Selbstverständlichkeit etablieren, werden auch von anderen Expert_innen beschrieben. Sie unterstreichen insbesondere die Bedeutung persönlicher Kontakte. Sich gegenseitig zu kennen und miteinander das Gespräch miteinander zu suchen, sei der erste Schritt für eine fruchtbare Zusammenarbeit, die stark von den einzelnen Personen und deren Engagement abhänge. Der Wille zur Zusammenarbeit stehe für einen Paradigmenwechsel in der Urbanisierungspolitik und die Gründung des Vereins unterstreiche das.

Drittens bildete paradoxerweise gerade die Zerschnittenheit der bestehenden Regulierungen in der Metropolitanregion Zürich eine entscheidende Grundlage für den erfolgreichen Aufbau des Vereins. Gerade weil keine überkantonalen Strukturen bestehen, gibt es kaum Konflikte mit bereits vorhandenen Kompetenzen. Entgegen des nationalstaatlichen Ziels des räumlichen Ausgleichs scheinen die Akteur_innen im VMZ kaum Probleme mit der Anerkennung urbaner Zentren zu haben. Offenbar sind die Urbanisierungsprozesse in solchem Ausmass vom Einfluss Zürichs geprägt, dass die (gegenseitigen) Interdependenzen augenfällig sind:

Über den Support der Nachbarkantone habe ich gestaunt. Der Verein hat extrem schnell an Dynamik gewonnen. Insbesondere die kleineren Kantone waren ausschlaggebend. Sie machten mit und fanden es wichtig, dass sich der Metropolitanraum Zürich als Kraft in der Schweiz und gegenüber dem Ausland formiert. Sie sagten, dass sie sich auf Zürich ausrichten. Der Flughafen - alles sei in Zürich. Und wenn sie sich international verkaufen wollten, dann müsse Zürich im Namen vorkommen. Darum war der Name nie ein grosses Thema. Das hat mich wirklich erstaunt. (Interview 8, 2012)

Dieses Zitat verorte ich im neoliberalen Diskurs des internationalen Standortwettbewerbs. Die Zeitdiagnose der Scale-

\footnotetext{
${ }^{5}$ Richtpläne legen die Grundzüge der angestrebten räumlichen Entwicklung fest.
} 
Debatte bestätigend, soll die neue Struktur den Zugang zu Kapitalflüssen ermöglichen, der mit einer kleinräumigeren Organisation erschwert wäre. In diesem Fall soll insbesondere der Name Zürich diesen Zugang bieten. Gleichzeitig entbrannte im Frühjahr 2013 im Gemeinderat der Stadt Zürich eine Debatte um die Mitgliedschaft im Verein (Neuhaus, 2013). Vertreter_innen von bürgerlichen bis linken Parteien verlangten den Austritt aus dem Verein. Den Bürgerlichen ging es vor allem um die Senkung der öffentlichen Ausgaben; der rechts-konservativen Schweizerischen Volkspartei auch um den Verlust kommunaler Freiheit. Die links politisierenden Grünen hingegen stiessen die Austrittsdebatte an, weil sie in der verfolgten Verkehrspolitik des VMZ, die Autobahnen ausbauen will, eine weitere Förderung der Zersiedlung sehen. Die Grünen bemängeln aber auch den Meinungsbildungsprozess innerhalb des VMZ als ,undemokratisch“ (Interview 13, 2014), da nur ein Planungsbüro und Exekutivpolitiker_innen an den Diskussionen beteiligt seien, jedoch nicht die Bevölkerung. Der Entscheid im Gemeinderat fiel schlussendlich für den Verbleib im Verein. Die Diskussion zeigt aber, dass der Vermarktung des metropolitanen Raums für das urbane Zentrum wenig Bedeutung beigemessen wird. Die Stadt Zürich versucht sich gleichzeitig mit anderen Städten zu vernetzen, wie etwa im Städteverband und fordert beispielsweise ein stärkeres Stimmrecht für die Städte im Ständerat (Péclet, 2014).

\subsection{Subtile Macht des Vereinsrechts}

Auch wenn der Verein von den meisten Akteur_innen des Arrangements gestützt wird, stellt sich mit Blick auf die rechtliche Basis dennoch die Frage nach seiner Handlungsfähigkeit. Mit dem Vereinsrecht tangiert der neue Akteur die bestehenden Autonomien des schweizerischen Föderalismus nicht, was die Statuten des Vereins (Art. 5) explizit betonen. Um welche Form von grenzüberschreitender Zusammenarbeit kann es sich also tatsächlich handeln, wenn ihre rechtliche Basis lediglich auf dem Vereinsrecht beruht? Oder besteht die Macht gerade darin, ,nur“ ein Verein zu sein? Viele Interviewpartner_innen streben keine Institutionalisierung des Vereins an, weil das die „Akzeptanz sofort mindern würde" (Interview 9, 2012). Zudem ist der subtile Druck, den der Verein schafft, offenbar ausreichend um politische Entscheidungen der Kantone zu beeinflussen:

Man muss natürlich immer betonen, dass es ein Verein ist. Er hat absolut keine Berechtigung, föderalistische Strukturen aufzubrechen. Er kann nur die Zusammenarbeit optimieren und Identität schaffen, so dass die einzelnen Kantone und Gemeinden in diesem Raum zu denken beginnen. Und dass dann die Entscheidungen, die sie und nicht der Verein fällen können, den Interessen des Vereins entsprechen. (Interview 8, 2012)
Der Föderalismus und der Verein sollen sich also nicht in die Quere kommen, was bis anhin kaum ein Problem darstellt, weil im schweizerischen Föderalismus keine solche Massstabsebene besteht. Die Vereinsstruktur bietet zudem wesentliche Freiheiten. Diverse Interviewpartner_innen betrachten sowohl die relativ unkomplizierte Finanzierung von Projekten wie auch die direkte Kommunikation zwischen Gebietskörperschaften verschiedener Massstabsebenen als Erleichterung. Die Flexibilität im Vergleich zum regulierteren Arbeitsalltag im föderalistischen System wird geschätzt. Von „Experimentiercharakter“ (Interview 8, 2012) und „Ideenwerkstatt“" (Interview 7, 2012) ist die Rede.

Es hat seinen Reiz auf dieser riesigen Ebene zu arbeiten, wo es noch keine gefestigten Strukturen und Abläufe gibt. Das schafft Spielraum, ist eine Befreiung und macht Spass. (Interview 6, 2012)

Bis jetzt fand ich in der ganzen Organisation sehr schön, dass es keine Strukturen gab. Das war das Spannende. Man konnte machen, was man wollte. Man hat das Geld nicht einfach herausgeschmissen, aber man war viel flexibler. (...). Aber jetzt wird es formeller. (Interview 7, 2012)

Diese Offenheit kommt gerade durch die verhältnismässige Informalität des Vereinsrechts zustande und ermöglicht vieles, was in der formalen föderalistischen Struktur nicht durchführbar wäre. So finden seit kurzem Sitzungen der acht Kantonsplaner_innen statt. Weil der Austausch in den Regionalkonferenzen die Metropolitanregion Zürich zerschneidet, haben sich Workshops etabliert, die diese Lücke füllen sollen. Der Verein kann offensichtlich bereits als Legitimation für gemeinsames Handeln herangezogen werden:



Aus diesen informellen Treffen entstanden bereits sichtbare Raumproduktionen. Als Paradebeispiel gilt die gemeinsame Äusserung von 14 Kantonen der Ost- und Zentralschweiz 
während der Anhörung im Vorfeld der nationalen Abstimmung über Finanzierung und Ausbau der Bahninfrastruktur (FABI). Die Kantone priorisierten ihre Interessen auf zwei Hauptanliegen zur besseren Erschliessung der Stadt Zürich, den Zimmerbergtunnel (Verbindung nach Zug und Luzern) und den Brüttener Tunnel (Verbindung nach Winterthur) (Schaffner, 2012). Diese Forderungen unterstreichen erneut die Bedeutung der wirtschaftlichen Zentren, die mit der Bundespolitik, aber auch mit dem VMZ zusätzlich gestärkt werden sollen. Zweitens ist eine gemeinsame Äusserung sowohl für die beteiligten Kantone als auch für den Bund eine effizientere Politikform. Denn statt mindestens 14 verschiedene Anliegen werden bereits abgestimmte Interessen eingereicht, was den Kantonen wiederum höhere Berücksichtigung verspricht. Viele Interviewte bekräftigen, dass eine solche Interessensabstimmung nur dank dieser Treffen möglich gewesen sei. Dem Konsens, der in diesen Strukturen zwischen den acht Kantonen etabliert wurde, haben sich später sechs weitere Kantone angeschlossen. Die gemeinsame Äusserung führte zur Aufstockung des Bahninfrastruktur-Fonds von 3.5 auf 6.4 Milliarden Schweizer Franken (Metropolitankonferenz Zürich, 2013). In der Konsequenz bewilligten die Stimmberechtigten mit der Annahme der FABI-Vorlage im Februar 2014 einen weiteren räumlichen Kompromiss: Die Bahninfrastruktur soll sowohl in den Zentren als auch in den periphereren Räumen ausgebaut werden.

Das zweite Exempel ist das im VMZ entwickelte Metrobild. Es soll ,als Denkhilfe für eine gemeinsame Ausrichtung der räumlichen Entwicklungsstrategien dienen, damit die prägende Vielfalt erhalten und weiterentwickelt und gleichzeitig die Leistungsfähigkeit des Raums verstärkt wird“ (berchtoldkrass space\&options, 2011:17). In seiner Konsequenz soll das Metrobild zur Abstimmung der kantonalen Richtpläne führen - eine wesentliche Veränderung der konzeptionellen Produktion des Raums.

Beide Beispiele produzieren aber nicht nur einen neuen konzeptionellen Raum, sondern haben auch Einfluss auf den materiellen und den gelebten Raum, beispielsweise mit dem Ausbau der Bahnverbindungen. Sie brechen partiell auch die föderalistische Struktur auf - etwa mit dem Metrobild, das als metropolitanes Raumentwicklungskonzept dient - und können in den Worten der Leiterin des ARE als Schritte hin $\mathrm{zu}$ „Formen eines modernen Föderalismus“ (Lezzi, 2013) gelesen werden. Metropolitane Arrangements sollen keine vierte Staatsebene schaffen, aber mit ,partnerschaftliche[n] Formen der Zusammenarbeit (...) schnell eine gute Lösung (...) finden“" (ibid).

\section{Fazit: Informelle Räumlichkeit als Vorteil für den VMZ - widersprechende Positionen als Frage der Massstabsebene?}

Das Metrobild und die Äusserung zur FABI-Vorlage geben Aufschluss über die Bedeutungen des VMZ: Zum einen soll er anhand gemeinsamer Strategien die Sichtbarkeit des Metropolitanraums in der Bundespolitik erhöhen. Dieses Ziel war auch für die Gründung der Metropolitankonferenz Basel konstitutiv (Merki, 2012). Zum anderen wird der Verein durch Verhandlungen und schliesslich gemeinsamen Forderungen auch zum Dienstleister für Kantone, die Mühe haben, Massnahmen zur Siedlungsbegrenzung durchzusetzen (Interview 9, 2012). Die Existenz des Vereins kann zur Legitimierung von gemeinsamem Handeln, die Beschlüsse zur Legitimierung von Massnahmen herangezogen werden. Der Konsens und damit die Übermacht der sieben anderen Kantone üben erheblichen Druck auf kantonale Entscheide aus. Der VMZ ist ein eher informelles Arrangement, in dem Entscheide gefällt, oder vielleicht auch „bloss“ eine gemeinsame Sprache, gemeinsame Ideen und gemeinsame Sichtweisen auf Probleme entwickelt werden, die dann (in unterschiedlich davon abweichenden Formen) in Kantons- und Gemeindebehörden fliessen und so formalisiert werden. Auf Ebene der Metropolitanregion lassen sich gleichzeitig Informalisierungen und Flexibilisierungen, aber auch neue Formalisierungen beobachten, die zu einer schrittweisen Institutionalisierung der neuen Handlungsebene führen.

Unabhängig vom Massstab schafft sich grenzüberschreitende Zusammenarbeit, zumindest in der Schweiz, in der Regel neue Strukturen, weil sie früher deutlich seltener stattfand. Diese Gefüge etablieren sich hauptsächlich über informelle, flexible Strukturen, die (zumindest in einem ersten Schritt) nicht klaren gesetzlichen Anforderungen unterworfen sind. Ihre Krux ist, dass einerseits nicht öffentlich über ihre Ausgestaltung und Beteiligung gesprochen wird, sie anderseits Kooperationen und Vorgehensweisen ermöglichen, die gerade deshalb zu Stande kommen. Über die gefassten Beschlüsse wiederum gibt es kaum öffentliche Auseinandersetzungen, da sie legitimiert und formal scheinen. Die Zusammenarbeit ermöglicht eine abgestimmtere Steuerung der Urbanisierung, die etwa dem aktuellen gesellschaftlichen Leitbild der Begrenzung der Siedlungsfläche entgegenkommt. Kantone und Gemeinden scheinen bereit, einen gewissen Teil ihrer Autonomie abzugeben. Der Verein schafft - wenn auch noch schwach institutionalisiert - eine neue territoriale Ebene im föderalistischen System der Schweiz. Belina (2013) schlägt anhand der Metropolitanregion Frankfurt Rhein-Main den Begriff „Continuously Emergent Scalar Fix" vor, um mit der Vorstellung eines institutionalisierten Scalar Fix zu brechen. Es wird sich zeigen, wie die Institutionalisierung des VMZ in Zukunft verläuft und ob diese Bezeichnung weiterhin passend scheint.

Nicht nur Informalisierungs- und Formalisierungsprozesse sind miteinander verknüpft, sondern auch Inkludierung und Exkludierung. Am untersuchten Beispiel lässt sich eine deutliche Expertisierung der Politiken der Urbanisierung beobachten. Erstens vergibt der VMZ zahlreiche, eher konzeptionelle Aufträge an Planungsbüros und ähnliche Firmen, die mit ihren Arbeiten den Diskurs beeinflussen und für Aufträge gleichzeitig von solchen Organisationen abhängig sind 
- auch eine Form der Privatisierung. Zweitens zieht der Verein in seinen Arbeitsgruppen für die Diskussion von Projekten im „Echoraum“Expert_innen bei, die von den jeweiligen Projektleiter_innen ausgewählt werden.

Andere nichtstaatliche Akteur_innen, die nicht Teil des Arrangements sind, werden auf der metropolitanen Ebene nicht mit gegenläufigen Meinungen zur durchgesetzten Strategie sichtbar. Das, so meine These, hängt direkt mit der metropolitanen Massstabsebene zusammen, die im föderalistischen System der Schweiz nicht institutionalisiert ist. Die ,informelle Räumlichkeit“ des Vereins begünstigt die Durchsetzbarkeit von Projekten, da sich institutionalisierte Akteur_innen, etwa Verbände, nicht auf dem metropolitanen Massstab organisieren, sondern auf einer oder mehreren Ebenen des föderalistischen Systems, auf denen auch die politischen Mittel von Initiative und Referendum bestehen. Der grossräumige Massstab bewirkt gleichzeitig auch, dass sich schwächer organisierte Gruppen oder einzelne Personen kaum kritisch zu den Projekten äussern. Solcher Widerstand findet häufig auf Quartierebene statt (z.B. Mayer, 2007:97), wo Urbanisierungsprojekte für die Bewohner_innen oft direkter und konkreter fassbar sind als Diskurse und Konzepte. Die Massstabsebene ist entscheidend dafür, welche Akteur_innen auftreten. Gerade weil der VMZ eine neue Massstabsebene schafft, bietet er die Möglichkeit einer unumstrittenen Aushandlungsebene. Damit scheint der untersuchte Fall das Argument einer skalaren Verschiebung von mächtigen Arrangements auf einen grossräumigeren Massstab um Konflikte zu umgehen, zu bestätigen.

Die Analyse unterstützt auch das Argument, dass sich metropolitane Regulationsweisen herausbilden, um die Produktion des Raums der Geografie des Kapitals anzupassen. Die Kantone fühlen sich nicht in ihren Kompetenzen bedroht, sondern sehen im Verein (neben anderem) die Möglichkeit, sich als gemeinsamer Wirtschaftsraum zu vermarkten. Ökonomische Argumente zur Begründung der Notwendigkeit des Vereins kommen aber nicht nur in den Interviews zum Tragen. Die Vermarktung der Metropolitanregion und damit der Kampf um Investitionsgelder ist auch Zweck des Vereins, der von einer neoliberalen Agenda geprägt ist (vgl. Kübler, 2013). Allerdings finden sich in den Interviews neben den neoliberalen Argumentationen auch einige wachstumskritische Narrative:

Die Grenzen des Wachstums sollten thematisiert werden (...). Der Flächenverbrauch pro Kopf steigt stetig. [...]. Es braucht eine Sensibilisierung der Bevölkerung oder vielleicht einmal eine richtige Depression. Der Flächenverbrauch hängt auch mit dem Wohlstand und dem Geld zusammen, das wir fürs Wohnen haben. (Interview 6, 2012)

Mag die Wettbewerbsfähigkeit für einige Akteur_innen der Hauptgrund für die Zusammenarbeit sein, so scheint sie für die meisten Interviewpartner_innen keine wichtige Rolle zu spielen. Das liegt sicher auch am Sampling, das hauptsächlich Personen mit einem raumplanerischen Hintergrund umfasst, die im grossräumigeren Denken eher eine Chance zur besseren Regulation der Urbanisierung sehen. Die wachstumskritische Haltung ist aber auch Ausdruck einer Paradigmenverschiebung. Einerseits wurde in einigen Interviews auf die kapitalistische Krise seit 2008 hingewiesen, die Wachstumsprozesse in Frage stelle. Statt noch mehr Wachstum und Wettbewerb sehen die meisten Interviewpartner_innen das starke ökonomische und bauliche Wachstum als zentrale Herausforderung der nächsten zehn Jahre und verlangen Beschränkungen des Wachstums sowie Wohnraum für einkommensschwächere Personen. Diese Forderungen werden teilweise auch von rechtskonservativen Kreisen geteilt, wie etwa die anfangs 2014 angenommene „Masseneinwanderungsinitiative" oder die im im Herbst 2014 abgelehnte „Ecopopinitiative“ zeigen. In beiden Fällen führen die Befürworter_innen raumpolitische Argumente ins Feld, um die Immigration restriktiver zu regulieren. Solche Forderungen sind auch Zeichen einer weiterhin wachsenden Finanzmetropole, die zunehmende Ungleichheit mit sich bringt und damit soziale Fragen im Raum, der immer auch Ausdruck von Machtverhältnissen ist, verschärft. Bereits wollen die Regierungen der Kantone Zug und Nidwalden, deren Urbanisierungsprozesse von der Tiefsteuerpolitik gezeichnet sind, Zonen für ,preisgünstigen Wohnraum“ schaffen (Aschwanden, 2014).

Die meist linken Forderungen nach günstigem Wohnraum und einer Beschränkung des räumlichen und wirtschaftlichen Wachstums sind aber nicht zuletzt auch durch kritische Diskurse und Praxen seitens widerständischer Formen wie Quartierkämpfe oder Recht-auf-Stadt-Gruppierungen sensibilisiert worden. Solche Bewegungen sind zwar kaum auf dem metropolitanen Massstab organisiert, doch thematisch über Analysen und Forderungen international vernetzt und damit durchaus auch auf dem metropolitanen Massstab gegenwärtig (vgl. Mayer, 2008). Vermutlich vermögen auch Diskurse der kritischen Stadtforschung zu dieser Präsenz beitragen.

Nichtsdestotrotz bleibt der VMZ bis anhin ein unumstrittenes, technokratisches Expert_innenbündnis. Vielleicht kommt es auch deshalb kaum zu Auseinandersetzungen um Struktur und Ziele des Vereins, weil die Institutionalisierung im Gewande des Vereins - einer urhelvetischen Form mit sozialem Anstrich - harmlos erscheint. Diese ,unschuldige“ Informalität begünstigt seine Wirkungsmacht. Der VMZ stellt bis heute eine ziemlich effiziente Steuerungsform dar, die hauptsächlich von staatlichen Akteur_innen geprägt wird und widerspruchslos erscheint. Diese Effizienz ist gerade darum möglich, weil die Restrukturierung auf einer neuen Massstabsebene und in vereinsrechtlicher Form stattfindet, was bis anhin viel Spielraum offen lässt. Es wird sich zeigen, wie lange die Produktion einer neuen Ebene und gemeinsamer Ziele so unumstritten fortgeführt werden kann.

Entgegen einiger Beiträge innerhalb der Scale-Debatte, die metropolitane Restrukturierungen als weitere Ermäch- 
tigung von Kapitalinteressen und der politischen Elite dechiffrieren, könnte eine Neuformierung auch eine neue Form von Demokratie und sozialen Kooperationsformen bedeuten (Ronneberger und Schmid, 1995:375-378). Swyngedouw (1997:159-161), aber auch Brenner und Heeg (1999:117) sind überzeugt, dass ein alternatives Urbanisierungsmodell nur mit überlokalen Mobilisierungen und Strategien erreicht werden kann. Während Solidarisierungen auf dem globalen Massstab durchaus stattfinden, kann zumindest auf Ebene der Metropolitanregion Zürich keine entsprechende politische Bewegung identifiziert werden. Die Frage, weshalb es kaum zu Auseinandersetzungen kommt, bleibt neben einigen Teilantworten, die ich hier skizziert habe, weiterhin offen.

Danksagung. Ich danke Peter-Paul Bänziger, Christian Schmid, Monika Streule sowie den zwei anonymen Referent_innen für ihre Kommentare zu verschiedenen Arbeitsständen dieses Artikels.

Edited by: B. Korf

Reviewed by: two anonymous referees

\section{Literatur}

Altrock, U., Bertram, G., und Fischer, F.: Urbane Governance in historischer Perspektive: Zur Konzeption des Bandes, in: Wer entwickelt die Stadt? Geschichte und Gegenwart lokaler Governance: Akteure - Strategien - Strukturen, Herausgeber: Altrock, U. und Bertram, G., transcript, Bielefeld, 7-25, 2012.

Aschwanden, E.: Nicht zum „Monaco der Schweiz“, Neue Zürcher Zeitung, 53, 13, 5 März 2014.

Bassand, M.: La métropolisation de la Suisse, Presse polytechniques et universitaire romandes, Lausanne, 2004.

Bassand, M. und Schuler, M.: La Suisse, une métropole mondiale? Rapport de recherche 54, IREC, EPFL, Lausanne, 1985.

Behrendt, H. and Kruse, C.: Die europäische Metropolregion Zürich : die Entstehung des subpolitischen Raumes, Geogr. Helv., 56, 202-213, doi:10.5194/gh-56-202-2001, 2001.

Belina, B.: Skalare Praxis, in: Politics of Scale. Räume der Globalisierung und Perspektiven emanzipatorischer Politik, Herausgeber: Wissen, M., Röttger, B. und Heeg, S., Westfälisches Dampfboot, Münster, 106-123, 2008.

Belina, B.: Region, Strukturierte Kohärenz und Continuously Emergent Scalar Fix. Zu Kooperation und Konkurrenz in de Global City Region Frankfurt Rhein-Main, Herausgeber: Brand, O., Dörhöfer, S. und Eser, P., Westfälisches Dampfboot, Münster, 140$161,2013$.

Belina, B., Naumann, M. und Strüver, A.: Handbuch Kritische Stadtgeographie, Westfälisches Dampfboot, Münster, 2014.

Benz, A.: Multilevel Governance - Governance in Mehrebenensystemen, in: Governance - Regieren in komplexen Regelsystemen. Eine Einführung, Herausgeber: Benz, A. und Dose, N., VS Verlag für Sozialwissenschaften, Wiesbaden, 111-135, 2010.

Benz, A. und Dose, N.: Governance - Modebegriff oder nützliches sozialwissenschaftliches Konzept? in: Governance - Regieren in komplexen Regelsystemen. Eine Einführung, Herausgeber: Benz, A. und Dose, N., VS Verlag für Sozialwissenschaften, Wiesbaden, 13-36, 2010. berchtoldkrass space\&options: Metrobild. Ein Bild für den Metroraum Zürich, pdf unter www.metropolitanraum-zuerich.ch (Zugriff: 10 Februar 2014), 2011.

Blanc, J.: Planlos in die Zukunft? Zur Bau- und Siedlungspolitik in den 50er Jahren, in: achtung: die 50er Jahre! Annäherungen an eine widersprüchliche Zeit, Herausgeber: Blanc, J. und Luchsinger, C., Chronos, Zürich, 71-93, 1994.

Blöchlinger, H.: Baustelle Föderalismus. Metropolitanregionen versus Kantone. Untersuchungen und Vorschläge für eine Revitalisierung der Schweiz, Herausgeber: Avenir Suisse, Verlag Neue Zürcher Zeitung, Zürich, 2005.

Brenner, N.: Between fixity and motion: accumulation, territorial organization and the historical geography of spatial scales, Environ. Plann. D, 16, 459-482, 1998.

Brenner, N.: New State Spaces. Urban Governance and the Rescaling of Statehood, Oxford University Press, Oxford, 2004.

Brenner, N.: What Is Critical Urban Theory? City, 13, 198-207, 2009.

Brenner, N. und Heeg, S.: Lokale Politik und Stadtentwicklung nach dem Fordismus: Möglichkeiten und Beschränkungen, Kurswechsel, 2, 103-119, 1999.

Brenner, N. und Schmid, C.: The "Urban Age" in Question, Int. J. Urban Regional, 38, 731-755, 2014.

Brunnengräber, A. und Walk, H. (Hrsg.): Multi-Level-Governance. Klima-, Umwelt- und Sozialpolitik in einer interdependenten Welt, Nomos-Verlag, Baden-Baden, 2007.

Bundesamt für Raumentwicklung (ARE): Raumentwicklungsbericht 2005, ARE, Bern, 2005.

Bundesamt für Statistik (BFS): Historisiertes Gemeindeverzeichnis, http://www.portal-stat.admin.ch/gde-tool/core/xshared/ gewo.php?lng=de-de (Zugriff: 24 Februar 2014).

Castells, M. und Godard, F.: Monopolville: analyse des rapports entre l'entreprise, l'état et l'urbain à partir d'une enquête sur la croissance industrielle et urbaine de la région de Dunkerque, Mouton, Paris, 1974.

Diener, R., Herzog, J., Meili, M., Meuron, P. und de und Schmid, C.: Die Schweiz. Ein städtebauliches Portrait, Birkhäuser Verlag für Architektur, Basel, 2006.

Dzudzek, I.: Unternehmen oder Unvernehmen? - Über die Krise des Kreativsubjekts und darüber hinaus, Geogr. Helv., 68, 181-189, doi:10.5194/gh-68-181-2013, 2013.

Dzudzek, I. und Müller, M.: Der Lärm des Politischen. Die Londoner riots 2011 und ihre politischen Subjekte, sub $\backslash$ urban, 1, 1740, 2013.

Eidgenössische Steuerverwaltung (ESTV): Steuerbelastung in der Schweiz. Natürliche Personen nach Gemeinden 2012, BFS, Neuchâtel, 2013.

Einig, K., Grabher, G., Ibert, O. und Strubelt, W.: Urban Governance, Informationen zur Raumentwicklung, 9/10, I-IX, 2005.

Eisinger, A.: Städte bauen. Städtebau und Stadtentwicklung in der Schweiz 1940-1970, gta Verlag, Zürich, 2004.

Eisinger, A. und Schneider, M., Stadtland Schweiz. Untersuchungen und Fallstudien zur räumlichen Struktur und Entwicklung in der Schweiz, Birkhäuser, Basel, 2003.

Etzemüller, T.: Social engineering als Verhaltenslehre des kühlen Kopfes. Eine einleitende Skizze, in: Die Ordnung der Moderne. Social Engineering im 20. Jahrhundert, Herausgeber: Etzemüller, T., transcript, Bielefeld, 11-39, 2009. 
Füller, H. und Marquardt, N.: One step back to see the whole picture: conceiving governance as power relations, in: Urban Governance in Europe, Future Urban Research in Europe 2, Herausgeber: Eckardt, F. und Elander, I., Berliner Wissenschafts-Verlag, Berlin, 93-110, 2009.

Fürst, D.: Regional Governance, in: Governance - Regieren in komplexen Regelsystemen. Eine Einführung, Herausgeber: Benz, A. und Dose, N., VS Verlag für Sozialwissenschaften, Wiesbaden, 49-68, 2010.

Gomes de Matos, C.: Das Modell Barcelona-Partizipation, Protest und Postpolitik, sub \urban, 1, 121-140, 2013.

Hall, P. und Pain, K. (Hrsg.): The polycentric metropolis: Learning from mega-city regions in Europe, Earthscan, London, 2006.

Hall, T. und Hubbard, P. (Hrsg.): The Entrepreneurial City, Wiley, Chichester, 1998

Harvey, D.: The urbanization of capital, Blackwell, Oxford, 1985.

Harvey, D.: From Managermialism to Entrepreneurialism: The Transformation in urban Governance in late Capitalism, Geografiska Annaler, 71, 3-17, 1989.

Heeg, S.: Politische Regulation des Raums: Metropolen, Regionen, Nationalstaat, Sigma, Berlin, 2001.

Heinelt, H. und Kübler, D.: Metropolitan governance in the 21st century: Capacity, democracy and the dynamics of place, Routledge, London, 2005.

Hermann, M. und Leuthold, H.: Atlas der politischen Landschaften. Ein weltanschauliches Porträt der Schweiz, vdf, Zürich, 2003.

Herod, A.: The production of scale in the United States labour relations, Area, 23, 82-88, 1991.

Herod, A. und Wright, M.: Placing Scale: An Introduction, in: Geographies of Power. Placing Scale, Herausgeber: Herod, A. und Wright, M., Blackwell, Oxford, 1-14, 2002.

Interview 1: Expert_in einer Nicht-Regierungsorganisation, 12 Oktober 2011.

Interview 2: Expert_in eines staatlichen Transportunternehmens, 20 April 2012.

Interview 3: Expert_in eines kantonalen Planungsamts, 23 April 2012.

Interview 4: Expert_in einer Nicht-Regierungsorganisation, 24 April 2012.

Interview 5: Expert_in einer Nicht-Regierungsorganisation, 2 März 2012.

Interview 6: Expert_in eines kantonalen Planungsamts, 8 März 2012.

Interview 7: Expert_in eines kantonalen Planungsamts, 10 März 2012.

Interview 8: Expert_in einer Nicht-Regierungsorganisation, 21 März 2012.

Interview 9: Expert_in eines kantonalen Planungsamts, 6 Juni 2012. Interview 10: Expert_in des nationalen Planungsamts, 31 Juli 2012.

Interview 11: Expert_in eines kantonalen Planungsamts, 27 November 2012 .

Interview 12: Expert_in einer Nicht-Regierungsorganisation, 3 Juni 2013.

Interview 13: Expert_in einer politischen Partei, 17 Juli 2014.

Jessop, B.: The rise of governance and the risks of failure: the case of economic development, Int. Soc. Sci. J., 50, 29-45, 1998.

Jessop, B.: Multi-Level Governance and Multi-Level Metagovernance. Changes in the European Union as Integral Moments in the Transformation and Reorientation of Contemporary State- hood, Herausgeber: Bache, I. und Flinder, M., Oxford University Press, Oxford, 49-74, 2004.

Jessop, B.: The European Union and recent transformations in statehood, in: The State of Europe: Transformations of Statehood from a European Perspective, Herausgeber: Puntscher Riekmann, S., Mokre, M., und Latzer, M., Campus, Frankfurt, 75-94, 2004.

Jessop, B.: Avoiding Traps, Rescaling States, Governing Europe, in: Leviathan Undone? Herausgeber: Keil, R. und Mahon, R., UBC Press, Vancouver, 87-104, 2009.

Jessop, B. und Sum, N.: An Entrepreneurial City in Action: Hong Kong's Emerging Strategies in and for (Inter)Urban Competition, Urban Stud., 37, 2287-2313, 2000.

Jonas, A.: Editorial, Environ. Plann. D, 12, 257-264, 1994.

Kemper, J. und Vogelpohl, A.: Zur Konzeption kritischer Stadtforschung. Ansätze jenseits einer Eigenlogik der Städte, sub $\backslash$ urban, 1, 7-30, 2013 .

Kilminster, R.: Critique and overcritique in sociology, Human Figurations 2, http://hdl.handle.net/2027/spo.11217607.0002.205, 2013.

Kipfer, S.: Die Zentralität des Städtischen: Überlegungen zur Skalenfrage, in: Politics of Scale. Räume der Globalisierung und Perspektiven emanzipatorischer Politik, Herausgeber: Wissen, M., Röttger, B., und Heeg, S., Westfälisches Dampfboot, Münster, 85-105, 2008.

Koll-Schretzenmayr, M.: Gelungen - misslungen? Die Geschichte der Raumplanung Schweiz, NZZ Libro, Zürich, 2008.

Krätke, S.: Europas Stadtsystem zwischen Metropolisierung und Globalisierung, Berlin, LIT, 2007.

Krebs, H.: Fordismus: Entwicklung und Krise - Eine Grobskizze, in: Unternehmen Globus. Facetten nachfordistischer Regulation, Herausgeber: Bruch, M. und Krebs, H., Westfälisches Dampfboot, Münster, 11-39, 1996.

Kübler, D.: Introduction: Metropolitanisation and metropolitan governance, Eur. Polit. Sci., 11, 402-408, 2012.

Kübler, D.: Vers une nouvelle gouvernance territoriale des espaces métropolitains en Suisse? L'example de la métropole zurichoise, in: Les horizons de la gouvernance territoriale, Herausgeber: Vodoz, L., Thévoz, L. und Faure, P., 105-115, 2013.

Kübler, D. und Koch, P: Re-scaling network governance. The evolution of public transport management in two Swiss agglomerations, Flux, 72, 108-119, 2008.

Kübler, D. und Scheuss, U.: New developments in Swiss metropolitan policy: the lost dimension found, in: Governing metropolises. Profiles of issues and experiments on four continents, Herausgeber: Collin, J. und Robertson, M., Presses de l'Université Laval, Montréal, 165-186, 2007.

Kübler, D., Scheuss, U., und Rochat, P.: The Metropolitan Bases of Political Cleavage in Switzerland, in: The political ecology of the metropolis, Herausgeber: Sellers, J. M., Kübler, D., WalterRogg, M. und Walks, A. R., EPCR Press, Colchester, 2013.

Kuster, J. und Meier, H. R.: Metropolitanraum Zürich, Porträt, Metropolitankonferenz Zürich, 2008.

Leendertz, A.: Ordnung, Ausgleich, Harmonie. Koordinaten raumplanerischen Denkens in Deutschland, 1920 bis 1970, in: Die Ordnung der Moderne. Social Engineering im 20. Jahrhundert, Herausgeber: Etzemüller, T., transcript, Bielefeld, 129-150, 2009. 
Lefebvre, H.: Die Revolution der Städte, List, München, 1972 [1970].

Lefebvre, H.: The production of space, Blackwell, Oxford, 1991 [1974].

Leitner, H. und Sheppard, E.: The Spatiality of Contentious Politics: More than Politics of Scale, in: Leviathan Undone? Herausgeber: Keil, R. und Mahon, R., UBC Press, Vancouver, 231-245, 2009.

Lemke, T.: Foucault, Governmentality, and Critique, Rethinking Marxism, 14, 49-64, 2002.

Lendi, M.: Raumplanung: ihr politischer Stellenwert in einer veränderten Welt. Angedacht am Beispiel der Schweiz, an einem Sonderfall? Raumforschung und Raumordnung, 66, 383-397, 2008.

Lezzi, M.: „Ein Raumkonzept kann auch auf Ebene Metropolitanraum erstellt werden.“, http: //www.metropolitanraum-zuerich.ch/lebensraum/ stossrichtung-lebensraum/interview-mit-maria-lezzi.html, Zugriff: 1 März 2013.

Lipietz, A.: Der Regulationsansatz, die Krise des Kapitalismus und ein alternativer Kompromiss für die neunziger Jahre, in: Capitales Fatales. Urbanisierung und Politik in den Finanzmetropolen Frankfurt und Zürich, Herausgeber: Hitz, H., Keil, R., Lehrer, U., Ronneberger, K., Schmid, C. und Wolff, R., Rotpunktverlag, Zürich, 90-122, 1995.

Mahon, R. und Keil, R.: Introduction, in: Leviathan Undone? Herausgeber: Keil, R. und Mahon, R., UBC Press, Vancouver, 3-23, 2009.

Marston, S. A., Jones, J. P. und Woodward, K.: Human geography without scale, T. I. Brit. Geogr., 30, 416-432, 2005.

Massey, D.: For space, SAGE Publications, London, 2005.

Mayer, M.: Stadtpolitik im Umbruch, in: Capitales Fatales. Urbanisierung und Politik in den Finanzmetropolen Frankfurt und Zürich, Herausgeber: Hitz, H., Keil, R., Lehrer, U., Ronneberger, K., Schmid, C. und Wolff, R., Rotpunktverlag, Zürich, 123-136, 1995.

Mayer, M.: Contesting the Neoliberalisation of Urban Governance, in: Contesting neoliberalism: urban frontiers, Herausgeber: Leitner, H., Peck, J. und Sheppard, E., Guilford, New York, 90-115, 2007.

Mayer, M.: Multiskalare Praxen städtischer Bewegungen, in: Politics of Scale. Räume der Globalisierung und Perspektiven emanzipatorischer Politik, Herausgeber: Wissen, M., Röttger, B. und Heeg, S., Westfälisches Dampfboot, Münster, 268-289, 2008.

Mayntz, R. und Scharpf, F. W.: Steuerung und Selbstorganisation in staatsnahen Sektoren, in: Gesellschaftliche Selbstregulierung und politische Steuerung, Herausgeber: Mayntz, R. und Scharpf, F. W., Campus, Frankfurt, 9-38, 1995.

Mayntz, R.: Governance im modernen Staat, in: Governance - Regieren in komplexen Regelsystemen, Herausgeber: Benz, A., VS Verlag für Sozialwissenschaften, Wiesbaden, 65-76, 2004.

McCann, E.: "Best Places": Interurban Competition, Quality of Life and Popular Media Discourse, Urban Stud., 41, 1909-1929, 2004.

McCann, E. und Ward, K.: Relationality/territoriality: toward a conceptualization of cities in the world, Geoforum, 41, 175-184, 2010.

Merki, M.: Mehr Einfluss in Bern nehmen, Neue Zürcher Zeitung, 13, 13, 17 Janur 2012.

Metropolitankonferenz Zürich 2013: Verkehrskommission, Medienmitteilung,
fileadmin/user_upload/downloads/Medienmitteilung_FABI_ Metropolitankonferenz_130219.pdf, 19 Februar 2013.

Miller, B.: Is Scale a Chaotic Concept? Notes on the Processes of Scale Production, in: Leviathan Undone?, Herausgeber: Keil, R. und Mahon, R., UBC Press, Vancouver, 51-66, 2009.

Neuhaus, C.: Metropole will Metropolitanraum verlassen, Neue Zürcher Zeitung, 119, 11, 27 May 2013.

Nüssli, R.: Between farming villages and hedge fund centres: politics of urbanisation in the border zone of the metropolitan region of Zurich, to be published, 2015.

Paasi, A.: Regions are "social constructs" but who or what constructs them? Agency in question, Environ. Plann. A, 42, 22962301, 2010.

Peck, J.: Political Economies of Scale: Fast Policy, Interscalar Relations, and Neoliberal Workfare, Econ. Geogr., 78, 331-360, 2002.

Péclet, J.: "Les grandes villes devraient avoir un siège au Conseil des Etats", Le Matin Dimanche, 17, 13 April 2014.

Plüss, L.: Die Kantonsgrenze im Metropolitanraum Zürich: Eine Untersuchung der interkommunalen Zusammenarbeit in der Nutzungsplanung, disP, 44, 66-76, 2008.

Przyborski, A. und Wohlrab-Sahr, M.: Qualitative Sozialforschung: Ein Arbeitsbuch, Oldenbourg Wissenschaftsverlag, München, 2009.

Rancière, J.: Disagreement?, politics and philosophy, University of Minnesota Press, Minneapolis, 1999.

Rhodes, R.: Understanding Governance: Policy Networks, Governance, Reflexivity and Accountability, Open University Press, Buckingham, 1997.

Ronneberger, K. und Schmid, C.: Globalisierung und Metropolenpolitik: Überlegungen zum Urbanisierungsprozess der neunziger Jahre, in: Capitales Fatales. Urbanisierung und Politik in den Finanzmetropolen Frankfurt und Zürich, Herausgeber: Hitz, H., Keil, R., Lehrer, U., Ronneberger, K., Schmid, C. und Wolff, R., Rotpunktverlag, Zürich, 354-378, 1995.

Rosemann, T.: Planning in the Face of Democracy. Mit Jacques Rancière über Raumplanung und Demokratie nachdenken, sub $\backslash$ urban, 1, 41-60, 2013.

Rosenau, J. N.: Strong Demand, Huge Supply: Governance in an Emerging Epoch, in: Multi-level Governance, Herausgeber: Blache, I. und Flinders, M., Oxford University Press, Oxford, 31-48, 2004.

Rosol, M.: On resistance in the post-political city: conduct and counter-conduct in Vancouver, Space and Polity, 18, 70-84, 2014.

Sack, D.: Urbane Governance, in: Handbuch Stadtsoziologie, Herausgeber: Eckardt, F., Springer, Wiesbaden, 311-335, 2012.

Sassen, S.: The global city: New York, London, Tokyo, Princeton University Press, Princeton, 1991.

Schaffner, D.: Zürich kämpft mit Ost- und Zentralschweiz für mehr Bahn, Tages-Anzeiger, 4, 28 März 2012.

Scheuplein, C.: Massstabsebenen der Regulation, in: Politics of Scale. Räume der Globalisierung und Perspektiven emanzipatorischer Politik, Herausgeber: Wissen, M., Röttger, B. und Heeg, S., Westfälisches Dampfboot, Münster, 186-207, 2008.

Schmid, C.: Raum und Regulation. Henri Lefebvre und der Regulationsansatz, in: Fit für den Postfordismus? Theoretischpolitische Perspektiven des Regulationsansatzes, Westfälisches Dampfboot, Münster, 217-242, 2003. 
Schmid, C.: Stadt, Raum und Gesellschaft. Henri Lefebvre und die Theorie der Produktion des Raumes, Franz Steiner, Stuttgart, 2005.

Schmid, C.: Theorie, in: Die Schweiz. Ein städtebauliches Portrait, Herausgeber: Diener, R., Herzog, J., Meili, M., de Meuron, P. und Schmid, C.: Birkhäuser Verlag für Architektur, Basel, 163223, 2006.

Schmid, C.: Wie gross ist Zürich? Anmerkungen zu einer aktuellen Debatte, SEES/Revue économique et sociale, 4, 67-82, 2007.

Schuler, M., Dessemontet, P. und Joye, D.: Die Raumgliederung der Schweiz, BFS, Neuchâtel, 2005.

Schweizerischer Bundesrat: Raumkonzept Schweiz, Überarbeitete Fassung, Bern, 2012.

Scott, A. J.: Regional motors of the global economy, Futures, 28, 391-411, 1996.

Scott, A. J., Agnew, J., Soja, E., und Storper, M.: Global CityRegions, in: Global City-Regions, Herausgeber: Scott, A. J., 1130, 2001.

Smith, N.: Uneven Development. Nature, Capital and the Production of Space, Blackwell, Oxford, 1984.

Smith, N.: Homeless/global: Scaling places, in: Mapping the futures - Local cultures, global change, Herausgeber: Bird, J., Curtis, B., Putnam, T., Robertson, G. und Tickner, L., Routledge, London, 87-119, 1993.

Smith, N.: Remaking scale: competition and cooperation in prenational and postnational Europe, in: Competitive European Peripheries, Herausgeber: Eskelinen, H. und Snickars, F., Springer, Berlin, 59-74, 1995.

Somerville, P.: Community governance and democracy, Policy Polit., 33, 117-144, 2005.

Storper, M.: The regional world: territorial development in a global economy, Guilford Press, New York, 1997.

Strauss, A. und Corbin, J.: Grounded Theory. Grundlagen qualitativer Sozialforschung, Beltz, Weinheim, 1996 [1990].

Sturm, R. und Bauer, M.: Governance und Regionen - die theoretische Debatte, in: Regional Governance in EU-Staaten, Herausgeber: Dieringer, J. und Sturm, R., Budrich, Opladen, 11-36, 2010.

Swyngedouw, E.: The heart of the place. The resurrection of locality in an age of hyperspace, Geographiska Annaler, 71, 31-42, 1989.

Swyngedouw, E.: The Mammon quest; "Glocalization", interspatial competition and the monetary order: The construction or new scales, in Cities and regions in the new Europe, Herausgeber: Dunford, M. und Kafkalas, G., Belhaven press, London, 39-66, 1992.

Swyngedouw, E.: Neither Global nor Local. "Glocalization" and the politics of scale, in: Spaces of Globalization. Reasserting the Power of the Local, Herausgeber: Cox, K. R., Guilford Press, New York, 137-166, 1997.
Swyngedouw, E.: The Post-Political City, in: Urban Politics Now. Re-imagining democracy in the neoliberal city, Herausgeber: BAVO, NAi Publishers, Rotterdam, 58-67, 2007.

Swyngedouw, E.: The Zero-Ground of Politics: Musings on the Post-Political City, in: Urban Asymmetries. Studies and Projects on Neoliberal Urbanization, Herausgeber: Kaminer, T., RoblesDurán, M. und Sohn, H., 010 Publishers, Rotterdam, 22-33, 2010.

Swyngedouw, E., Moulaert, F. und Rodriguez, A.: Neoliberal Urbanization in Europe: Large-Scale Urban Development Projects and the New Urban Policy, in: Spaces of Neoliberalism. Urban Restructuring in North America and Western Europe, Herausgeber: Brenner, N. und Theodore, N., Blackwell, Oxford, 195-229, 2004.

Taylor, P.: A materialist framework for political geography, T. I. Brit. Geogr., 7, 15-34, 1982.

Taylor, P.: World City Network. A global urban analysis, Routledge, London, 2004.

Thierstein, A., Kruse, C., Glanzmann, L., Gabi, S. und Grillon, N.: Raumentwicklung im Verborgenen : die Entwicklung der Metropolregion Nordschweiz, Verlag Neue Zürcher Zeitung, Zürich, 2006.

van der Heiden, N., Koch, P. und Kübler, D.: Rescaling metropolitan governance: examining discourses and conflicts in two Swiss metropolitan areas, Urban Research and Practice, 6, 40-53, 2013.

Veltz, P.: Mondialisation, villes et territoires. L'économie d'archipel, PUF, Paris, 1996.

Wissen, M.: Politics of Scale. Multi-Level Governance aus der Perspektive kritischer (Raum-)Theorien, in: Multi-LevelGovernance. Klima-, Umwelt- und Sozialpolitik in einer interdependenten Welt, Herausgeber: Brunnengräber, A. und Walk, H., Nomos-Verlag, Baden-Baden, 2007.

Wissen, M.: Zur räumlichen Dimensionierung sozialer Prozesse. Die Scale-Debatte in der angloamerikanischen Radical Geography - eine Einleitung, in: Politics of Scale. Räume der Globalisierung und Perspektiven emanzipatorischer Politik, Herausgeber: Wissen, M., Röttger, B. und Heeg, S., Westfälisches Dampfboot, Münster, 8-32, 2008.

Wissen, M., Röttger, B. und Heeg, S. (Hrsg.): Politics of Scale. Räume der Globalisierung und Perspektiven emanzipatorischer Politik, Westfälisches Dampfboot, Münster, 2008.

Zimmermann, K. und Heinelt, H.: Metropolitan Governance in Deutschland. Das Regieren in Ballungsräumen und neue Formen der politischen Steuerung, Springer, Wiesbaden, 2012.

Žižek, S.: Some Politically Incorrect Reflections on Urban Violence in Paris and New Orleans and Related Matters, Herausgeber: BAVO, NAi Publishers, Rotterdam, 12-29, 2007. 